\section{HLA discrepancy between graft and host rather than that graft and first donor impact the second transplant outcome}

\author{
Yoshinobu Maeda, ${ }^{1^{*}}$ Tomotaka Ugai, ${ }^{2,3^{*}}$ Eisei Kondo, ${ }^{4}$ Kazuhiro Ikegame, ${ }^{5}$ \\ Makoto Murata, ${ }^{6}$ Naoyuki Uchida, ${ }^{7}$ Toshihiro Miyamoto, ${ }^{8}$ Satoshi Takahashi, ${ }^{9}$ \\ Kazuteru Ohashi, ${ }^{10}$ Hirohisa Nakamae, ${ }^{11}$ Takahiro Fukuda, ${ }^{12}$ \\ Makoto Onizuka, ${ }^{13}$ Tetsuya Eto, ${ }^{14}$ Shuichi Ota, ${ }^{15}$ Makoto Hirokawa, ${ }^{16}$ \\ Tatsuo Ichinohe, ${ }^{17}$ Yoshiko Atsuta, ${ }^{18}$ Yoshinobu Kanda ${ }^{3,19}$ and Junya Kanda; ${ }^{20}$ \\ on behalf of the HLA Working Group of the Japan Society for Hematopoietic \\ Cell Transplantation
}

${ }^{1}$ Department of Hematology and Oncology, Okayama University Graduate School of Medicine, Dentistry and Pharmaceutical Sciences; ' 2 Division of Cancer Epidemiology and Prevention, Department of Preventive Medicine, Aichi Cancer Center Research Institute, Nagoya; ${ }^{3}$ Division of Hematology, Saitama Medical Center, Jichi Medical University, Tochigi; ${ }^{4}$ Division of Hematology, Department of Medicine, Kawasaki Medical School, Okayama; ${ }^{5}$ Division of Hematology, Department of Internal Medicine, Hyogo Medical College; ${ }^{6}$ Department of Hematology and Oncology, Nagoya University Graduate School of Medicine; ${ }^{7}$ Department of Hematology, Federation of National Public Service Personnel Mutual Aid Associations Toranomon Hospital, Tokyo; ${ }^{8} \mathrm{Hematology}$, Oncology \&

Cardiovascular medicine, Kyushu University Hospital, Fukuoka; ${ }^{9}$ Division of Molecular Therapy, The Advanced Clinical Research Center, The Institute of Medical Science, The University of Tokyo; ${ }^{10} \mathrm{Hematology}$ Division, Tokyo Metropolitan Cancer and Infectious Diseases Center, Komagome Hospital, Tokyo; ${ }^{11}$ Department of Hematology, Osaka City University Hospital; ${ }^{12}$ Department of Hematopoietic Stem Cell Transplantation, Nationa Cancer Center Hospital, Tokyo; ${ }^{13}$ Department of Hematology/Oncology, Tokai University School of Medicine, Kanagawa; ${ }^{14}$ Department of Hematology, Hamanomachi Hospital, Fukuoka; ${ }^{15}$ Department of Hematology, Sapporo Hokuyu Hospital, Hokkaido; ${ }^{16}$ Department of General Internal Medicine and Clinical Laboratory Medicine, Akita University Graduate School of Medicine; ${ }^{17}$ Department of Hematology and Oncology, Research Institute for Radiation Biology and Medicine (RIRBM), Hiroshima University; ${ }^{18}$ Department of Healthcare Administration, Nagoya University Graduate School of Medicine; ${ }^{19}$ Division of Hematology, Department of Medicine, Jichi Medical University, Tochigi and ${ }^{20}$ Department of Hematology and Oncology, Graduate School of Medicine, Kyoto University, Japan

*YM and TU contributed equally to this work.

\section{ABSTRACT}

econd allogeneic hematopoietic stem cell transplantation is a cura-

Stongen tive treatment option for patients with hematologic malignancies. However, it is unclear whether HLA discrepancy between graft and first donor has an impact on the outcome of second transplantation. We retrospectively analyzed 646 patients receiving second transplantation after an initial HLA mismatched transplantation. With regard to graft-versus-host, the one-allele mismatch (1 mismatch) group (SHR, 1.88; 95\%CI: $0.79-4.45 ; P=0.163)$ and more than one-allele mismatch group ( $\geq 2$ mismatch) (SHR, 1.84; 95\% CI, 0.75-4.51; $P=0.182$ ) had higher risks of grade III-IV acute graft-versus-host disease (GvHD) compared to the HLA-matched (0 mismatch) group. In contrast, no difference in risk of acute GvHD was found among the 0,1 , and $\geq 2$ mismatch group with respect to graft-versus-first donor. With regard to graft-versus-host, the $\geq 2$ mismatch group showed a significantly higher risk of treatmentrelated mortality (SHR, 1.90; 95\%CI, 1.04-3.50; $P=0.038$ ) compared to the 0 mismatch group, while the risk of relapse was slightly lower in the $\geq 2$ mismatch group (SHR, 068; 95\% CI, 0.44-1.06; $P=0.086$ ). In contrast, with regard to graft-versus-first donor, there were no significant differences in treatment-related mortality or relapse among the three groups. These findings suggested that HLA discrepancy between graft and host induces transplant-related immunological responses in second transplantation leading to an increase in treatment-related mortality, in contrast, the biological effects of HLA discrepancy between graft and first donor on outcome may be negligible.
Ferrata Storti Foundation

Haematologica 2019

Volume 104(5):1055-1061

\section{Correspondence:}

YOSHINOBU MAEDA

yosmaeda@md.okayama-u.ac.jp

Received: August 20, 2018.

Accepted: November 23, 2018.

Pre-published: December 6, 2018.

doi:10.3324/haematol.2018.204438

Check the online version for the most updated information on this article, online supplements, and information on authorship \& disclosures: www.haematologica.org/content/104/5/1055

\section{(C)2019 Ferrata Storti Foundation}

Material published in Haematologica is covered by copyright. All rights are reserved to the Ferrata Storti Foundation. Use of published material is allowed under the following terms and conditions:

https://creativecommons.org/licenses/by-nc/4.0/legalcode. Copies of published material are allowed for personal or internal use. Sharing published material for non-commercial purposes is subject to the following conditions:

https://creativecommons.org/licenses/by-nc/4.0/legalcode, sect. 3. Reproducing and sharing published material for commercial purposes is not allowed without permission in writing from the publisher. 


\section{Introduction}

For patients with malignant hematologic diseases who relapse after allogeneic hematopoietic stem cell transplantation (HSCT), a second HSCT is thought to be a curative option. It is believed that use of a second donor may confer increased therapeutic potency by inducing a more potent graft-versus-leukemia (GvL) effect; however, there are no data to support this assumption. ${ }^{1.9}$ In early studies, a second HSCT after a first HLA-matched transplantation was associated with similar risks of relapse and acute graft-versus-host disease (GvHD) using a different HLAmatched donor. ${ }^{3}$ There was no significant difference in survival between the transplantations from the original donor and another donor.

Over the years, the use of HLA-mismatched (MM) transplantation for hematologic diseases has increased, including haploidentical HSCT and cord blood transplantation (CBT). Following HLA-MM transplantation, a second donor is selected due to HLA discrepancy between the graft and the host. Physicians pay little attention to HLA discrepancy between the graft and the first donor, although the impact of this discrepancy on the outcome of second HSCT is unclear. Recipient non-hematopoietic gastrointestinal cells can express MHC class II, which is critical for inducing experimental acute GvHD in cases of minor histocompatibility antigen (mHAg) MM ${ }^{10,11}$ In contrast, hematopoietic antigen presenting cells (APCs), especially dendritic cells, induce MHC class I-dependent acute GvHD in mHAg MM cases. ${ }^{12}$ Furthermore, in the MHC MM setting, hematopoietic APCs play an important role in the induction of both MHC class I- and II-dependent acute GvHD..$^{13-16}$ As hematopoietic APCs are of first donor origin, HLA discrepancy between the graft and the first donor may be related to transplant-related immunological responses of the second HSCT.

To elucidate the biological effects of HLA discrepancy between the graft and the first donor that impact the outcome of the second HSCT, we compared the effects of HLA-MM between the graft and the first donor to those between the graft and the host in 646 patients receiving a second HSCT after an initial HLA-MM transplantation.

\section{Methods}

\section{Study population}

Patients who were at least 16 years of age with acute myelogenous leukemia (AML), acute lymphoblastic leukemia (ALL), myelodysplastic syndrome (MDS), chronic myelogenous leukemia (CML), malignant lymphoma (ML), or other malignant hematologic disease, and who received a second HSCT after an initial HLA-MM transplantation, were included in this study. Furthermore, patients must have received first and second allogeneic HSCTs between 1994 and 2016, with full HLA-A, -B, and -DRB1 allele data. Hematopoietic stem cell transplantation recipient clinical data were collected by the Japan Society for Hematopoietic Cell Transplantation (JSHCT) and the Japanese Data Center for Hematopoietic Cell Transplantation (JDCHCT) using the Transplant Registry Unified Management Program (TRUMP).17-19 We excluded individuals who: 1) first received HLA-matched HSCT; 2) received a second HSCT within 30 days after the first HSCT, in a planned manner or due to rejection/engraftment failure; 3) died within 30 days and lacked data on survival status and survival date; 4) lacked accurate allele data; or 5) received more than two HSCTs. The final study population consisted of 646 patients. The study was approved by the Data Management Committee of TRUMP and the Institutional Review Board of Okayama University.

\section{Study end points}

The outcomes assessed included acute GvHD, chronic GvHD, neutrophil engraftment, transplant-related mortality (TRM), relapse, and overall survival (OS). Acute and chronic GvHD were diagnosed and graded using the standard criteria. ${ }^{20,21}$ Neutrophil engraftment was considered to have occurred when the absolute neutrophil count was $\geq 0.5 \times 10^{9}$ cells/L for 3 consecutive days. Death from any cause was the event of interest in determining OS. TRM was defined as death during remission.

\section{Statistical analysis}

Descriptive statistics were generated for patients' characteristics. Differences in characteristics between groups were evaluated by the $\chi^{2}$ test and analysis of variance. The probability of OS was estimated according to the Kaplan-Meier method, and groups were compared using the log rank test. Subsequently, the probabilities of relapse, TRM, and acute and chronic GvHD were estimated on the basis of cumulative incidence curves. ${ }^{22}$ Competing events were death without relapse for relapse, relapse for TRM, death without engraftment for engraftment, and death without GvHD for acute or chronic GvHD. The groups were compared using Gray's test.

To evaluate the impact of HLA discrepancy on transplant outcomes, we estimated the hazard ratios (HRs) or subhazard ratios (SHRs) and 95\% confidence intervals (CIs) adjusted for potential confounders. The Cox proportional hazards model was used to evaluate the impact on OS, whereas multivariable competingrisks regression was used to evaluate the impact on the other end points. Several potential confounders considered in the multivariable analyses were provided in the Online Supplementary Appendix.

In all analyses, $P<0.05$ was considered statistically significant. All statistical analyses are performed with Stata (v.15,0; Stata Corp., College Station, TX, USA) and EZR software (Saitama Medical Center, Jichi Medical University, Japan). ${ }^{23}$

\section{Results}

\section{Patients' and transplantation characteristics}

A total of 646 patients who received a second HSCT after an initial HLA-MM transplantation were analyzed. Patients' and transplantation characteristics are presented in Table 1. With respect to the HLA discrepancy in the graft-versus-host direction (graft vs. host), HLA matching was categorized as follows: HLA -A, -B, -DRB1 match (0 $\mathrm{MM}, \mathrm{n}=85), \mathrm{MM}$ at one allele (1 MM, $\mathrm{n}=160)$, or mismatch at more than one allele ( $\geq 2 \mathrm{MM}, \mathrm{n}=401)$. With regard to HLA discrepancy in the graft-versus-first donor direction (graft $v s$. first donor), the second HSCT was categorized as follows: HLA -A, -B, -DRB1 match (0 MM, $\mathrm{n}=72$ ), mismatch at one allele ( $1 \mathrm{MM}, \mathrm{n}=100)$, or mismatch at more than one allele ( $\geq 2 \mathrm{MM}, \mathrm{n}=474)$. In the graft-versus-host comparison, the $\geq 2 \mathrm{MM}$ group received cord blood more frequently (0 MM, 20.0\%; $1 \mathrm{MM}, 21.3 \%$; $\geq 2 \mathrm{MM}, 60.2 \%, P<0.001)$, were more likely to use a reduced-intensity conditioning regimen (0 MM, 56.5\%; 1 $\mathrm{MM}, 66.3 \%$; $\geq 2 \mathrm{MM}, 70.7 \%, P=0.012)$, and had a higher rate of in vivo T-cell depletion (0 MM, 10.6\%; $1 \mathrm{MM}$, $18.1 \%$; $\geq 2 \mathrm{MM}, 25.4 \%, P=0.003)$. The interval between the first and second HSCT was shorter in this group $(<12$ 
Table 1. Patients' and donor characteristics.

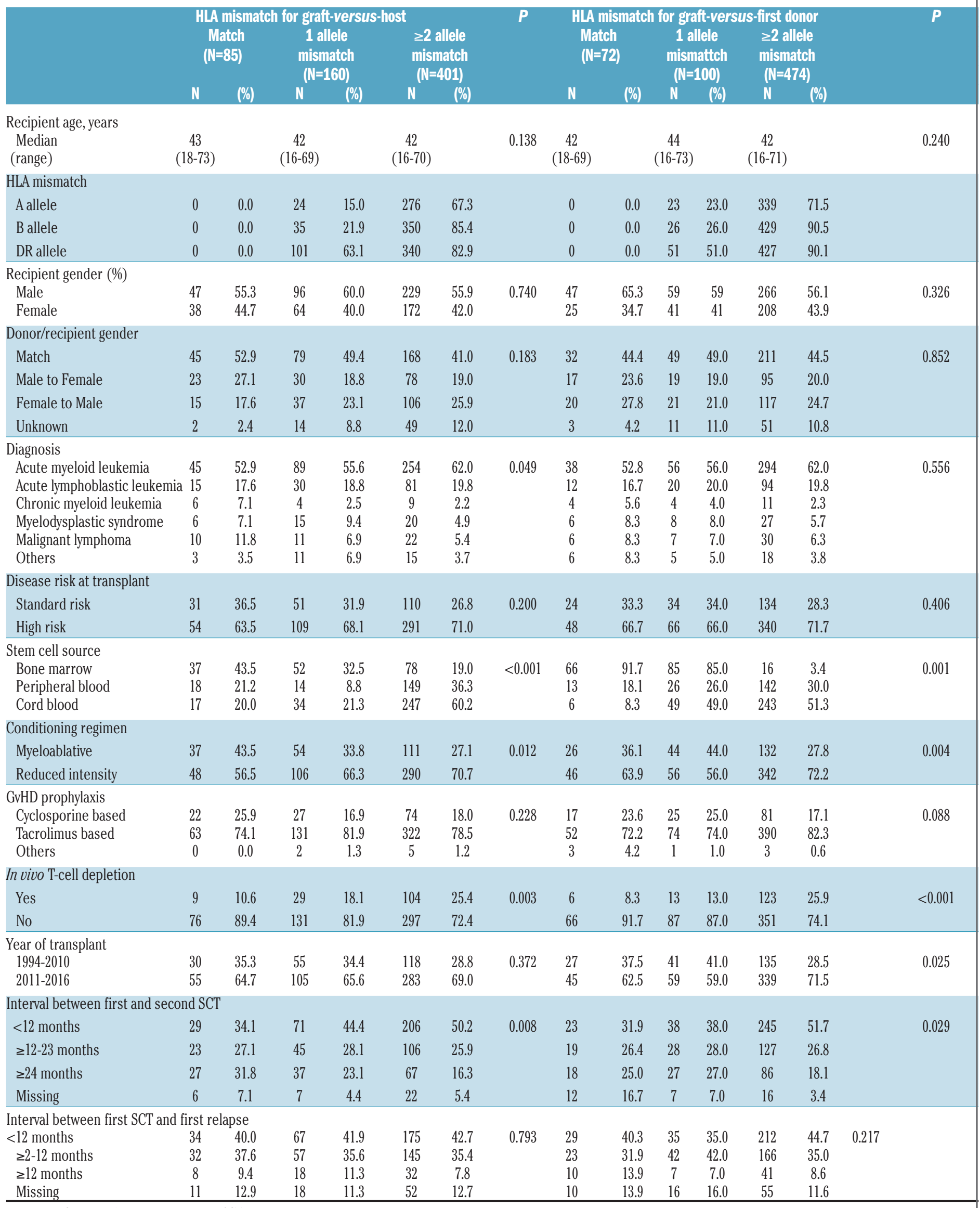

N: number; GvHD: graft-versus-host disease; SCT: stem cell transplantation. 
Table 2. Effect of HLA allele mismatch on acute graft-versus-host disease (GvHD), chronic GvHD and engraftment in multivariate analyses.

\begin{tabular}{|c|c|c|c|c|c|}
\hline \multirow{2}{*}{$x^{2}$} & \multicolumn{2}{|c|}{ HLA mismatch for graft-versus-host } & \multicolumn{3}{|c|}{ HLA mismatch for graft-versus-first donor } \\
\hline & $\begin{array}{l}1 \text { allele } \\
\text { mismatch } \\
(\mathrm{N}=160)\end{array}$ & $\begin{array}{c}\geq 2 \text { allele } \\
\text { mismatch } \\
(N=401)\end{array}$ & $\begin{array}{l}\text { Match } \\
(\mathrm{N}=72)\end{array}$ & $\begin{array}{l}1 \text { allele } \\
\text { mismatch } \\
(N=100)\end{array}$ & $\begin{array}{l}\geq 2 \text { allele } \\
\text { mismatch } \\
(N=474)\end{array}$ \\
\hline
\end{tabular}

Grades III to IV acute GvHD

$\mathrm{SHR}^{1}(95 \% \mathrm{CI})$

1 (ref)

1.88

1.84

1 (ref)

0.84

0.91

$(0.79-4.45, P=0.163) \quad(0.75-4.51, P=0.182)$

$(0.35-2.02, P=0.669) \quad(0.43-1.93, P=0.800)$

Chronic GvHD

$\mathrm{SHR}^{1}(95 \% \mathrm{CI})$

1 (ref)

1.45

1.20

1 (ref)

0.98

0.91

(0.84-2.50, $P=0.181)$

$(0.60-2.38, P=0.605)$

$(0.55-1.76, P=0.956)$

(0.54-1.51, $P=0.702)$

Neutrophil engraftment

$\mathrm{SHR}^{1}(95 \% \mathrm{CI})$

1 (ref)

0.81

0.77

1 (ref)

1.06

1.23

$(0.62-1.06, P=0.126) \quad(0.56-1.05, P=0.097)$

$(0.75-1.48, P=0.753) \quad(0.92-1.66, P=0.167)$

${ }^{1}$ Adjusted for recipient age at transplant (continuous), recipient gender, gender mismatch (match, male to female, female to male, unknown), diagnosis (acute myeloid leukemia, acute lymphoblastic leukemia, chronic myeloid leukemia, myelodysplastic syndrome, malignant lymphoma or others), disease risk at transplant (standard or high), stem cell source (bone marrow, peripheral blood, cord blood), conditioning regimen (myeloablative or reduced intensity), graft-versus-host disease (GvHD) prophylaxis (cyclosporine based, tacrolimus based, others), in vivo T-cell depletion (Yes, No), year of transplant (1994-2010,2011-2016), interval between first and second stem cell transplantation (SCT) ( $<12$ months, $\geq 12-23$ months, $\geq 24$ months, missing) and interval between first SCT and relapse ( $<2$ months, $\geq 2-12$ months, $\geq 12$ months, missing). SHR: subdistribution hazard ratios.

months: $0 \mathrm{MM}, 34.1 \%$; $1 \mathrm{MM}, 44.4 \%$; $\geq 2 \mathrm{MM}, 50.2 \%$, $P=0.008)$. With regard to graft-versus-first donor comparison, the $\geq 2 \mathrm{MM}$ group showed a similar trend to that for the graft-versus-host group comparison. The $\geq 2 \mathrm{MM}$ group required more cord blood $(P=0.001)$, used a reduced-intensity conditioning regimen $(P=0.004)$, and had greater in vivo $\mathrm{T}$-cell depletion $(P<0.001)$ and a shorter interval between the first and second HSCT $(P=0.029)$.

\section{Acute graft-versus-host disease, chronic graft-versus-host disease, and engraftment}

With regard to the graft-versus-host results, the unadjusted cumulative incidence rates of grade III-IV acute GvHD at 100 days post transplantation were 9.5\% (95\% CI: 4.4$17.0 \%$ ) in the 0 MM group, $13.8 \%$ (95\% CI: $9.0-19.7 \%$ ) in the $1 \mathrm{MM}$ group, and $11.0 \%$ (95\% CI: $8.2-14.3 \%$ ) in the $\geq 2$ $\mathrm{MM}$ group (Figure 1). In multivariate analysis, the $1 \mathrm{MM}$ group (SHR, 1.88; 95\%CI: 0.79-4.45; $P=0.163$ ) and $\geq 2 \mathrm{MM}$ group (SHR, 1.84; 95\%CI: 0.75-4.51; $P=0.182$ ) tended to have higher risk of grade III-IV acute GvHD compared to the $0 \mathrm{MM}$ group, although the results were not statistically significant (Table 2). With regard to affected organ, the risk of skin, gut and liver acute GvHD increased among the $1 \mathrm{MM}$ group and $\geq 2 \mathrm{MM}$ group compared to the $0 \mathrm{MM}$ group (Table 3 ). There was no statistically significant difference in risk of chronic GvHD among the groups in multivariate analysis. The cumulative incidence rate of neutrophil engraftment at day 50 was $94.0 \%$ (95\% CI: 85.6-97.6\%) in the $0 \mathrm{MM}$ group, $96.9 \%$ (95\%CI: $92.3-$ $98.7 \%$ ) in the $1 \mathrm{MM}$ group, and $91.0 \%$ (95\% CI: 87.7 $93.4 \%$ ) in the $\geq 2 \mathrm{MM}$ group. In multivariate analysis, the $\geq 2 \mathrm{MM}$ group tended to show delayed engraftment compared to the $0 \mathrm{MM}$ group (SHR, 0.77; 95\% CI: 0.56-1.05; $P=0.097)$.

With regard to the graft-versus-first donor results, there were no significant differences in the risk of grade III-IV acute GvHD, chronic GvHD, or neutrophil engraftment among the groups in multivariate analysis (Table 2).

Next, the association of each HLA allele MM with
GvHD was evaluated (Online Supplementary Table S1). With regard to graft-versus-host, B allele MM was associated with an increased risk of grade III-IV acute GvHD in multivariate analysis (SHR, 2.87; 95\% CI: 1.42-5.79; $P=0.003$ ), and $\mathrm{DR}$ allele $\mathrm{MM}$ was associated with delayed neutrophil engraftment (SHR, 0.80; 95\% CI: 0.67-0.95, $P=0.011)$; no such associations were found for the other $\mathrm{MM}$ types. With regard to the graft-versus-first donor results, no HLA allele MM showed an association with grade III-IV acute GvHD, chronic GvHD, or neutrophil engraftment in multivariate analysis.

\section{Transplant-related mortality, relapse, and overall survival}

With regard to the graft-versus-host results, the unadjusted cumulative incidence rates of TRM and relapse at 5 years post transplantation were 19.8\% (95\%CI: 11.8 $29.2 \%$ ) and $55.6 \%$ (95\%CI: 43.9-65.7\%) in the $0 \mathrm{MM}$ group, $32.5 \%$ (95\% CI:25.2-39.9\%) and $45.2 \%(95 \% \mathrm{CI}$ : $37.3-52.8 \%$ ) in the 1 MM group, and $34.7 \%$ (95\% CI: 30.0 $39.4 \%$ ) and $46.8 \%$ (95\% CI: $41.8-51.7 \%$ ) in the $\geq 2 \mathrm{MM}$ group, respectively (Figures 2 and 3). Multivariate analysis indicated that the risk of TRM was marginally higher in the $1 \mathrm{MM}$ group (SHR, 1.67; 95\% CI: 0.94-2.98; $P=0.081$ ), and significantly higher in the $\geq 2 \mathrm{MM}$ group (SHR, 1.90; 95\% CI: $1.04-3.50 ; P=0.038)$, versus the $0 \mathrm{MM}$ group. In contrast, the risk of relapse was slightly lower in both the $1 \mathrm{MM}$ group (SHR, 073; 95\% CI: 0.50-1.07; $P=0.110$ ) and the $\geq 2$ MM group (SHR, 068; 95\% CI: 0.44-1.06; $P=0.086$ ). Consequently, no significant differences in OS were found among the three groups in multivariate analyses (Table 4). Analysis of each HLA allele MM revealed that only HLA $\mathrm{DR}$ allele $\mathrm{MM}$ was significantly associated with a lower risk of relapse (SHR, $0.75 ; 95 \% \mathrm{CI}: 0.58-0.95 ; P=0.018)$ and a higher risk of TRM (SHR, 1.44; 95\%CI: 1.03-2.00; $P=0.033$ ) (Online Supplementary Table S2). The main causes of TRM differed among the three groups. The rates of interstitial pneumonia, TMA, and especially acute GvHD 


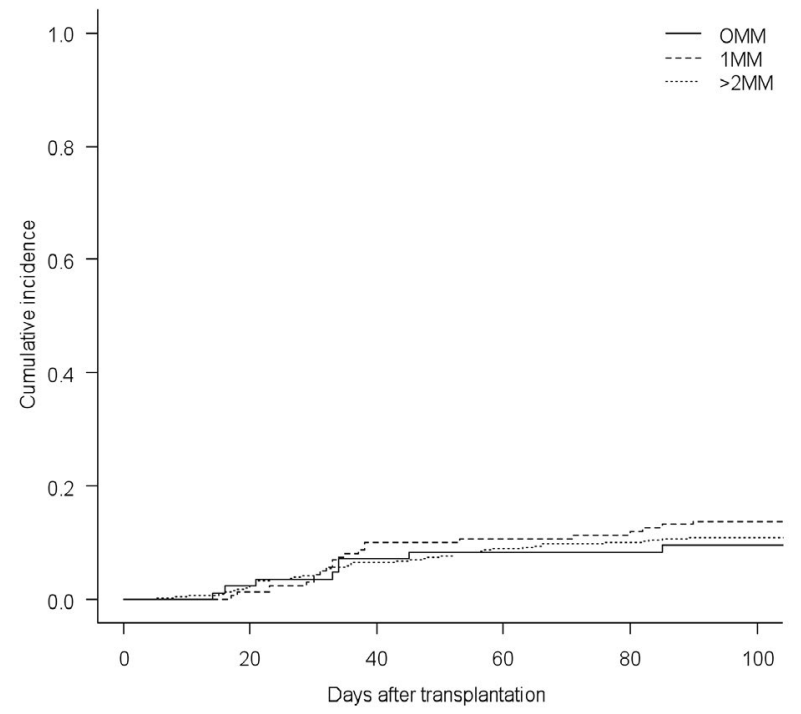

Figure 1. The unadjusted cumulative incidence of grades III to IV acute graft-versus-host disease (GvHD) by HLA mismatch (MM) for graft. With regard to the graft-versus-host results, the unadjusted cumulative incidence rates of grade III-IV acute GvHD were $9.5 \%(95 \% \mathrm{Cl}$ : $4.4-17.0 \%)$ in the $0 \mathrm{MM}$ group, $13.8 \%(95 \% \mathrm{Cl}: 9.0 \%-19.7 \%)$ in the $1 \mathrm{MM}$ group, and $11.0 \%(95 \% \mathrm{Cl}: 8.2 \%-$ $14.3 \%$ ) in the $\geq 2 \mathrm{MM}$ group.

(0 MM, 0.0\%; $1 \mathrm{MM}, 11.8 \%$; $\geq 2 \mathrm{MM} 10.9 \%$ ) were increased in the $1 \mathrm{MM}$ group and the $\geq 2 \mathrm{MM}$ group (Online Supplementary Table S3). With regard to the graft-versus-first donor outcomes, there were no significant differences in TRM, relapse, or OS among the three groups (Table 4). In addition, no allele MM was associated with relapse, TRM, or OS in the analysis of each HLA allele MM (Online Supplementary Table S2).

\section{Analyses by stem cell sources}

Finally, we performed analyses according to stem cell source (Online Supplementary Tables S4 and 5). We did not observe any obvious statistically heterogeneity among stem cell sources. However, the small sample size for some categories partially precluded evaluation of significance.

\section{Discussion}

There have been several studies on the role of donor change in the outcome of second HSCT; however, these studies were performed mainly in HLA-matched or $1 \mathrm{Ag}$ $\mathrm{MM}$ cases and focused on procedures in which a second HSCT from the same donor was performed. ${ }^{1-9}$ In this study, we evaluated the role of HLA discrepancy between the graft and host and between the graft and the first donor on the outcome of second HSCT after HLA-MM initial HSCT. On evaluating 646 recipients of a second HSCT, it was found that graft-host HLA-match was associated with a reduced rate of TRM compared to HLAMM, while HLA discrepancy between the graft and the first donor had no impact on the outcome of second HSCT.

In the largest retrospective analysis performed to date ( $n=1285$ patients) to compare the incidence of GvHD in the same cohort, the incidence rate of grade II-IV acute GvHD in first HSCT was $26 \%$ versus $46 \%$ in second $\mathrm{HSCT}^{24}$ In our study, the incidence of grade II-IV and

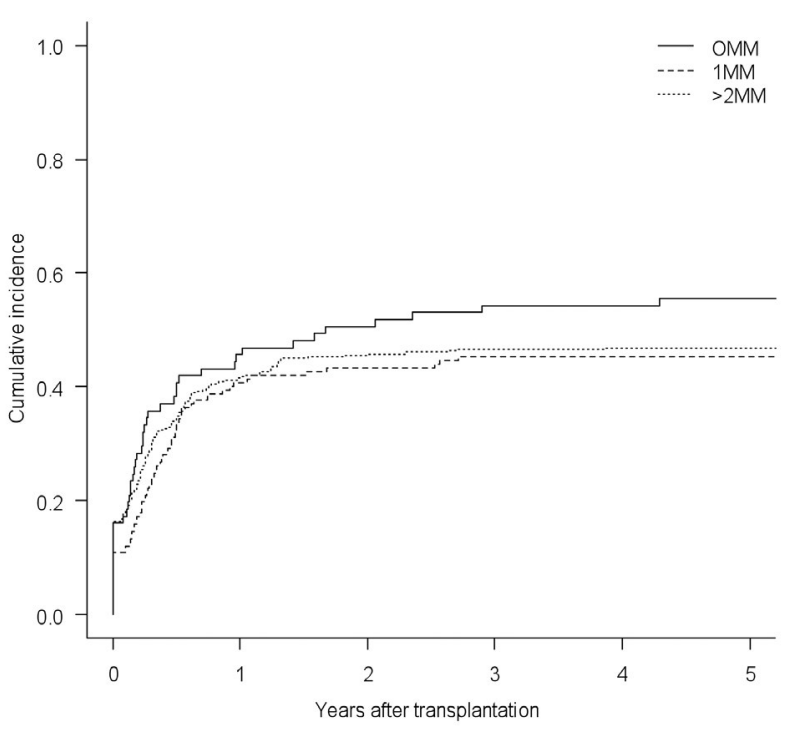

Figure 2. The unadjusted cumulative incidence of relapse by HLA mismatch (MM) for graft-versus-host. With regard to the graft-versus-host results, the unadjusted cumulative incidence rates of relapse were $55.6 \%(95 \% \mathrm{Cl}: 43.9$ $65.7 \%)$ in the $0 \mathrm{MM}$ group, $45.2 \%(95 \% \mathrm{Cl}: 37.3-52.8 \%)$ in the $1 \mathrm{MM}$ group, and $46.8 \%$ (95\% Cl: $41.8-51.7 \%$ ) in the $\geq 2$ MM group.

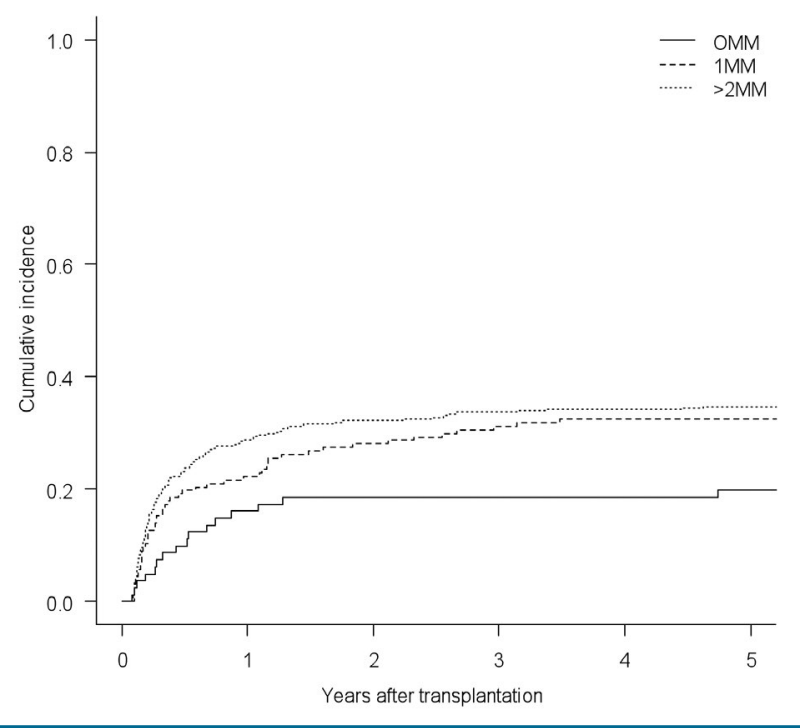

Figure 3. The unadjusted cumulative incidence of transplant-related mortality (TRM) by HLA mismatch (MM) for graft-versus-host. With regard to the graft-versus-host results, the unadjusted cumulative incidence rates of treatment-related mortality TRM were $19.8 \%(95 \% \mathrm{Cl}: 11.8-29.2 \%)$ in the $0 \mathrm{MM}$ group, $32.5 \%$ (95\% Cl: $25.2-39.9 \%)$ in the $1 \mathrm{MM}$ group, and $34.7 \%$ (95\% Cl: $30.0-39.4 \%)$ in the $\geq 2$ MM group.

grade III-IV acute GvHD for first HSCT was $36.4 \%$ and $9.0 \%$ versus $34.2 \%$ and $11.2 \%$, respectively, for second HSCT. Due to the higher rate of GvHD for second HSCT, prevention of acute GvHD represents an important, and as yet unmet, medical need.

Experimental murine studies reported that hematopoietic APCs play an important role in the induction of acute GvHD in an MHC MM setting. ${ }^{13-16}$

In the present study, HLA-MM between the graft and first donor was not associated with an increased risk of acute GvHD in HSCT recipients having hematopoietic 
Table 3. Effect of HLA allele mismatch on Grades III to IV acute graft-versus-host disease (GvHD) by affected organ.

$\begin{gathered}\text { HLA mismatch for graft-versus-host } \\ 1 \text { allele mismatch }(\mathrm{N}=160)\end{gathered} \quad \geq 2$ allele mismatch (N=401)

Skin GvHD

SHR1 (95\%CI)

1 (ref)

$2.49(0.87-7.13, P=0.088)$

$2.94(0.94-9.19, P=0.063)$

Gut GvHD

SHR1 (95\%CI)

1 (ref)

$3.33(0.90-12.3, P=0.072)$

$3.14(0.82-12.0, P=0.094)$

Liver GvHD

SHR1 (95\%CI)

1 (ref)

$2.16(0.53-8.85, P=0.283)$

$3.24(0.73-14.4, P=0.122)$

'Adjusted for recipient age at transplant (continuous), recipient gender, gender mismatch (match, male to female, female to male, unknown), diagnosis (acute myeloid leukemia, acute lymphoblastic leukemia, chronic myeloid leukemia, myelodysplastic syndrome, malignant lymphoma or others), disease risk at transplant (standard or high), stem cell source (bone marrow, peripheral blood, cord blood), conditioning regimen (myeloablative or reduced intensity), graft-versus-host disease (GvHD) prophylaxis (cyclosporine based, tacrolimus based, others), in vivo T-cell depletion (Yes, No), year of transplant (1994-2010,2011-2016), interval between first and second stem cell transplantation (SCT) ( $<12$ months, $\geq 12-23$ months, $\geq 24$ months, missing) and interval between first SCT and relapse ( $<2$ months, $\geq 2-12$ months, $\geq 12$ months, missing). SHR: subdistribution hazard ratios.

Table 4. Effect of HLA allele mismatch on transplant-related mortality, relapse and overall survival in multivariate analyses.

\begin{tabular}{|c|c|c|c|c|c|}
\hline \multirow[b]{2}{*}{$x^{2}$} & \multicolumn{2}{|c|}{ HLA mismatch for graft-versus-host } & \multicolumn{3}{|c|}{ HLA mismatch for graft-versus-first donor } \\
\hline & $\begin{array}{c}1 \text { allele } \\
\text { mismatch } \\
(\mathrm{N}=160)\end{array}$ & $\begin{array}{l}\geq 2 \text { allele } \\
\text { mismatch } \\
(\mathrm{N}=401)\end{array}$ & $\begin{array}{l}\text { Match } \\
(\mathrm{N}=72)\end{array}$ & $\begin{array}{l}1 \text { allele } \\
\text { mismatch } \\
N=100)\end{array}$ & $\begin{array}{l}\geq 2 \text { allele } \\
\text { mismatch } \\
(\mathrm{N}=474)\end{array}$ \\
\hline
\end{tabular}

Transplant-related mortality

SHR $^{1}(95 \% \mathrm{CI})$

1 (ref)

1.67

1.90

1 (ref)

0.89

0.67

$(0.94-2.98, P=0.081) \quad(1.04-3.50, P=0.038)$

$(0.52-1.52, P=0.665)$

$(0.42-1.07, P=0.095)$

\begin{tabular}{|c|c|c|c|c|c|c|}
\hline Relapse & & & & & & \\
\hline $\operatorname{SHR}^{1}(95 \% \mathrm{CI})$ & 1 (ref) & $\begin{array}{c}0.73 \\
(0.50-1.07, P=0.110)\end{array}$ & $\begin{array}{c}0.68 \\
(0.44-1.06, P=0.086)\end{array}$ & 1 (ref) & $\begin{array}{c}1.18 \\
(0.72-1.95, P=0.516)\end{array}$ & $\begin{array}{c}1.41 \\
(0.89-2.22, P=0.143)\end{array}$ \\
\hline Overall survival & & & & & & \\
\hline $\mathrm{HR}^{1}(95 \% \mathrm{CI})$ & 1 (ref) & $\begin{array}{c}1.00 \\
(0.72-1.41, P=0.952)\end{array}$ & $\begin{array}{c}1.21 \\
(0.84-1.73, P=0.313)\end{array}$ & 1 (ref) & $\begin{array}{c}0.84 \\
(0.57-1.21, P=0.347)\end{array}$ & $\begin{array}{c}0.85 \\
(0.61-1.17, P=0.313) \\
\end{array}$ \\
\hline
\end{tabular}

*Bold denotes statistical significance. ${ }^{1}$ Adjusted for recipient age at transplant (continuous), recipient gender, gender mismatch (match, male to female, female to male, unknown), diagnosis (acute myeloid leukemia, acute lymphoblastic leukemia, chronic myeloid leukemia, myelodysplastic syndrome, malignant lymphoma or others), disease risk at transplant (standard or high), stem cell source (bone marrow, peripheral blood, cord blood), conditioning regimen (myeloablative or reduced intensity), graft-versus-host disease (GvHD) prophylaxis (cyclosporine based, tacrolimus based, others), in vivo T-cell depletion (Yes, No), year of transplant (1994-2010, 2011-2016), interval between first and second stem cell transplantation (SCT) ( $<12$ months, $\geq 12-23$ months, $\geq 24$ months, missing) and interval between first SCT and relapse $(<2$ months, $\geq 2-12$ months, $\geq 12$ months, missing). SHR: subdistribution hazard ratios; HR: hazard ratios.

APCs originating from the first donor. The antigen-presenting function of the first-donor hematopoietic cells may be insufficiently strong to induce GvHD. An alternative explanation is that recipient hematopoietic APCs have a limited capacity to induce acute GvHD, possibly owing to their predisposition to induce donor T-cell death. ${ }^{11}$ In contrast, HLA discrepancy between the graft and host may impact the risk of acute GvHD during the second transplant. In this study, HLA-MM between the graft and host showed increased risk of grade III-IV acute GvHD, although the results were not significant. In addition, B allele MM was significantly associated with an increased risk of grade III-IV acute GvHD in the analysis of each HLA allele mismatch [relative risk (RR) 2.87, 95\% CI: 1.42-5.79; $P=0.003$ ]. Several experimental studies showed that non-hematopoietic gastrointestinal cells are able to express MHC class II and induce $\mathrm{CD}^{+}$ T-cell-dependent acute GvHD. ${ }^{10,11}$ As the antigen-presenting function of epithelial cells is enhanced in the presence of an inflammatory environment, epithelial cells after the first HSCT could play a major role in inducing GvHD following second HSCT, although further studies are needed to validate this.

The length of remission after first HSCT and the disease status at second HSCT, are two main independent prognostic factors for predicting the outcome of a second HSCT. ${ }^{2,3,5}$ Despite a significant increase in the proportion of patients of advanced age, having an advanced disease stage, and receiving alternative donor transplants, there has been a continual decrease in TRM, reflecting the impact of advances in supportive care and more widespread use of reduced-intensity conditioning regimens. However, the reduction in rate of TRM has been less obvious in patients following a second remission or refractory disease..$^{25}$ Due to more advanced disease and accumulating toxicity, second transplants are more problematic than first transplants, and often result in an increase in TRM and overall mortality rates. Attempted enhancement of the GvT effect by switching donor may be affected by the toxicity of the second HSCT. Reducing TRM remains one of the most significant challenges in second HSCT. Our analysis showed that HLA-MM between the graft and first donor had no influence on GvHD, relapse, TRM, or OS. In contrast, with regard to graft-versus-host, the risk of TRM was significantly higher in the $\geq 2 \mathrm{MM}$ group versus the $0 \mathrm{MM}$ group (RR, 1.90; 95\% CI: 1.04-3.50; $P=0.038$ ). Analysis of each HLA allele MM revealed that the DR allele MM was significantly associated with a lower rate 
of relapse versus the $0 \mathrm{MM}$ group (RR, 0.75 ; $95 \% \mathrm{CI}$ : $0.58-$ $0.95 ; P=0.018)$, but this was offset by a higher rate of TRM (RR, 1.44; 95\% CI: 1.03-2.00; $P=0.033$ ). Our data suggested that use of an HLA-MM donor may induce a more potent GvL effect, but also increases the allogeneic responses of the second HSCT and provokes an increase in TRM events. These effects tended to cancel each other out in respect to $\mathrm{OS}$.

This is the first study to focus on patients after initial HLA-MM transplantation and identify risk factors for a poor second HSCT outcome. However, several limitations of the study should be mentioned. First, although this was a relatively large-scale study on second transplant, the sample size was still modest, and therefore further studies with larger sample sizes are required. Second, it used a retrospective design and included a heterogeneous patient group. Moreover, the strategies of the different treatment centers with respect to donor change are unknown, and any heterogeneity in transplantation procedure, year of transplant, and patients' characteristics may have biased the results, although we attempted to reduce bias by adjusting for these factors in multivariate analyses. Third, we did not adjust for multiple comparisons and therefore caution is required when interpreting the results, in partic- ular those of the stratified analyses. In addition, HLA-C typing and high-resolution DNA typing were either rarely, or not routinely, performed on the donors. Finally, donor chimerism was not systematically analyzed and cell subset chimerism data were not available for most patients.

In conclusion, HLA-MM donor is an option after initial HLA-MM transplantation. However, TRM remains a challenge, particularly with $\mathrm{a} \geq 2 \mathrm{MM}$ donor regarding graft-versus-host. In this study, the biological effects of HLA discrepancy between the graft and the first donor on the outcome appeared negligible, and our findings shed light on the role of non-hematopoietic APCs on transplant-related immunological responses.

\section{Funding}

This work was supported in part by the Practical Research Project for Allergic Diseases and Immunology (Research Technology of Medical Transplantation) from Japan Agency for Medical Research and Development, AMED under Grant Number 18ek0510023h0002. The authors are grateful to all physicians and data managers at the centers who contributed valuable data on transplantation to the JMDP and TRUMP. The authors also thank the members of the data management committees of JDMP and TRUMP for their assistance.

\section{References}

1. Bosi A, Laszlo D, Labopin M, et al. Second allogeneic bone marrow transplantation in acute leukemia: results of a survey by the European Cooperative Group for Blood and Marrow Transplantation. J Clin Oncol. 2001;19(16):3675-3684

2. Shaw BE, Mufti GJ, Mackinnon S, et al. Outcome of second allogeneic transplants using reduced-intensity conditioning following relapse of haematological malignancy after an initial allogeneic transplant. Bone Marrow Transplant. 2008;42(12):783-789.

3. Eapen M, Giralt SA, Horowitz MM, et al. Second transplant for acute and chronic leukemia relapsing after first HLA-identical sibling transplant. Bone Marrow Transplant. 2004;34(8):721-727.

4. Christopeit M, Kuss O, Finke J, et al. Second allograft for hematologic relapse of acute leukemia after first allogeneic stem-cell transplantation from related and unrelated donors: the role of donor change. J Clin Oncol. 2013; 31(26):3259-3271

5. Ruutu T, de Wreede LC, van Biezen A, et al. Second allogeneic transplantation for relapse of malignant disease: retrospective analysis of outcome and predictive factors by the EBMT. Bone Marrow Transplant. 2015; 50(12):1542-1550

6. Vrhovac R, Labopin M, Ciceri F, et al. Second reduced intensity conditioning allogeneic transplant as a rescue strategy for acute leukaemia patients who relapse after an initial RIC allogeneic transplantation: analysis of risk factors and treatment outcomes. Bone Marrow Transplant. 2016;51(2):186-193.

7. Orti G, Sanz J, Bermudez A, et al. Outcome of Second Allogeneic hematopoietic cell transplantation after relapse of myeloid malignancies following allogeneic hematopoietic cell transplantation: a Retrospective Cohort on Behalf of the Grupo Espanol de Trasplante Hematopoyetico. Biol Blood Marrow
Transplant. 2016;22(3):584-588.

8. Andreola G, Labopin M, Beelen D, et al. Long-term outcome and prognostic factors of second allogeneic hematopoietic stem cell transplant for acute leukemia in patients with a median follow-up of 10 years. Bone Marrow Transplant. 2015;50(12):1508-1512.

9. Horstmann K, Boumendil A, Finke J, et al. Second allo-SCT in patients with lymphoma relapse after a first allogeneic transplantation. A retrospective study of the EBMT Lymphoma Working Party. Bone Marrow Transplant. 2015;50(6):790-794.

10. Jones SC, Murphy GF, Friedman TM, Korngold R. Importance of minor histocompatibility antigen expression by nonhematopoietic tissues in a CD4+ T cell-mediated graft-versus-host disease model. J Clin Invest. 2003;112(12):1880-1886.

11. Koyama M, Kuns RD, Olver SD, et al Recipient nonhematopoietic antigen-presenting cells are sufficient to induce lethal acute graft-versus-host disease. Nat Med. 2011; 18(1):135-142.

12. Shlomchik WD, Couzens MS, Tang CB, et al Prevention of graft versus host disease by inactivation of host antigen-presenting cells. Science. 1999;285(5426):412-415.

13. Teshima T, Ordemann R, Reddy $\mathrm{P}$, et al. Acute graft-versus-host disease does not require alloantigen expression on host epithelium. Nat Med. 2002;8(6):575-581

14. Duffner UA, Maeda Y, Cooke KR, et al. Host dendritic cells alone are sufficient to initiate acute graft-versus-host disease. J Immunol. 2004;172(12):7393-7398

15. Zhang Y, Louboutin JP, Zhu J, Rivera AJ Emerson SG. Preterminal host dendritic cells in irradiated mice prime CD8+ T cell-mediat ed acute graft-versus-host disease. J Clin Invest. 2002;109(10):1335-1344.

16. Reddy P, Maeda Y, Liu C, Krijanovski OI, Korngold R, Ferrara JL. A crucial role for antigen-presenting cells and alloantigen expression in graft-versus-leukemia responses. Nat
Med. 2005:11(11):1244-1249.

17. Atsuta Y, Suzuki R, Yoshimi A, et al. Unification of hematopoietic stem cell transplantation registries in Japan and establishment of the TRUMP System. Int J Hematol. 2007;86(3):269-274.

18. Atsuta $Y$. Introduction of Transplant Registry Unified Management Program 2 (TRUMP2) scripts for TRUMP data analyses, part I (variables other than HLA-related data). Int J Hematol. 2016;103(1):3-10.

19. Kanda J. Scripts for TRUMP data analyses Part II (HLA-related data): statistical analyses specific for hematopoietic stem cell transplantation. Int J Hematol. 2016;103(1):11-19.

20. Przepiorka D, Weisdorf D, Martin P, et al 1994 Consensus Conference on Acute GVHD Grading. Bone Marrow Transplant. 1995;15(6):825-828.

21. Sullivan KM, Agura E, Anasetti C, et al Chronic graft-versus-host disease and other late complications of bone marrow transplantation. Semin Hematol. 1991;28(3):250259.

22. Gooley TA, Leisenring W, Crowley J, Storer BE. Estimation of failure probabilities in the presence of competing risks: new representations of old estimators. Stat Med. 1999; 18(6):695-706.

23. Kanda Y. Investigation of the freely available easy-to-use software 'EZR' for medical statistics. Bone Marrow Transplant. 2013; 48(3):452-458.

24. Duncan CN, Majhail NS, Brazauskas R, et al Long-term survival and late effects among one-year survivors of second allogeneic hematopoietic cell transplantation for relapsed acute leukemia and myelodysplastic syndromes. Biol Blood Marrow Transplant. 2015;21(1):151-158.

25. Bacigalupo A, Sormani MP, Lamparelli T, et al. Reducing transplant-related mortality after allogeneic hematopoietic stem cell transplantation. Haematologica. 2004; 89(10):12381247. 